Paul H. Bezandry*

\title{
Almost periodic stochastic Beverton-Holt difference equation with higher delays and with competition between overlapping generations
}

https://doi.org/10.1515/msds-2020-0105

Received April 29, 2020; accepted October 5, 2020

Abstract: The paper studies the existence of an almost periodic solution of some system of stochastic Beverton-Holt equation with higher delays and with competition between overlapping generations under some reasonable assumptions.

Keywords: almost periodic sequence, Beverton-Holt difference equation, exponential dichotomy, time delay MSC: Primary 39A10, 60G07; Secondary 34F05

\section{Introduction}

Hereditary systems (or systems with delays or after-effects) are widely used to model processes in biology. Stochastic difference equations of order greater than one have received less studies about them, and they are imperative in application where the state (i.e the size of a population) after $k$ steps depends on the previous $l+1$ states $(l \geq 1)$. A very important aspect of the qualitative study of the solutions of stochastic difference equations is their almost periodicity.

For a study of almost periodic (or almost automorphic) sequences we refer the reader to (Bezandry and Diagana [2], Bezandry et al. [3], Corduneanu [4], Diagana [5], Diagana et al. [6], Han and Hong [8], Hong and Nunez [9]) and references therein. Almost periodicity is also of importance in the study of stochastic processes.

In this paper, we investigate the solutions of some system of stochastic Beverton-Holt equation with higher delays and with competition between overlapping generations given by

$$
X_{t+1}=\gamma_{t} X_{t}+f\left(t, X_{t-\tau}, X_{t-\tau+1}, \ldots, X_{t-1}, X_{t}\right), t \in \mathbb{Z}_{+}
$$

where $\gamma_{t} \in(0,1)$ is the survival rate for each $t \in \mathbb{Z}_{+}, \tau$ is a fixed positive integer, and $f: \mathbb{Z}_{+} \times \mathbb{R}^{\tau+1} \rightarrow \mathbb{R}_{+}$is the recruitment function given by

$$
f\left(t, X_{t-\tau}, X_{t-\tau+1}, \ldots, X_{t-1}, X_{t}\right)=\frac{\left(1-\gamma_{t}\right) \mu K_{t} X_{t}}{\left(1-\gamma_{t}\right) K_{t}+\left(\mu-1+\gamma_{t}\right)\left(\sum_{i=1}^{\tau+1} \beta_{i} X_{t-i+1}\right)},
$$

with $K_{t}>0$ being carrying capacity, $\beta_{i}$ 's are positive real numbers, and $\mu>1$ is the intrinsic growth rate.

We also assume that $K_{t}$ and $\gamma_{t}$ are random and that the $\gamma_{t}$ 's are independent and independent of $X_{0}$. This assumption together with Equation(1.1) imply that the sequence $\left\{\gamma_{t}\right\}_{t \in \mathbb{Z}_{+}}$is independent of the sequence $\left\{X_{t}\right\}_{t \in \mathbb{Z}_{+}} \cdot$

^Corresponding Author: Paul H. Bezandry: Department of Mathematics, Howard University, Washington, DC 20059, E-mail: pbezandry@howard.edu 
The paper is organized as follows. In Section 2, we recall a basic theory of almost periodic random sequences on $\mathbb{Z}_{+}$. In Section 3, we apply the techniques developed in Section 2 to find some sufficient conditions for the existence of almost periodic solutions to stochastic Beverton-Holt difference equation with higher delays and with competition between overlapping generations.

\section{Preliminaries}

In this section we establish a basic theory for almost periodic random sequences. To facilitate our task, we first introduce the notations needed in the sequel.

Let $(\mathbb{B},\|\cdot\|)$ be a Banach space and let $(\Omega, \mathcal{F}, \mathbf{P})$ be a complete probability space. Throughout the rest of the paper, $\mathbb{Z}_{+}$denotes the set of all nonnegative integers. Define $L^{1}(\Omega ; \mathbb{B})$ to be the space of all $\mathbb{B}$-valued random variables $V$ such that

$$
\mathbf{E}\|V\|:=\left(\int_{\Omega}\|V(\omega)\| d \mathbf{P}(\omega)\right)<\infty .
$$

It is then routine to check that $L^{1}(\Omega ; \mathbb{B})$ is a Banach space when it is equipped with its natural norm $\|\cdot\|_{1}$ defined by, $\|V\|_{1}:=\mathbf{E}\|V\|$ for each $V \in L^{1}(\Omega, \mathbb{B})$.

Let $X=\left\{X_{t}\right\}_{t \in \mathbb{Z}_{+}}$be a sequence of $\mathbb{B}$-valued random variables satisfying $\mathbf{E}\left\|X_{t}\right\|<\infty$ for each $t \in \mathbb{Z}_{+}$. Thus, interchangeably we can, and do, speak of such a sequence as a function, which goes from $\mathbb{Z}_{+}$into $L^{1}(\Omega ; \mathbb{B})$.

This setting requires the following preliminary definitions.

Definition 2.1. An $L^{1}(\Omega ; \mathbb{B})$-valued random sequence $X=\left\{X_{t}\right\}_{t \in \mathbb{Z}_{+}}$is said to be Bohr almost periodic in mean if for each $\varepsilon>0$ there exists $N_{0}(\varepsilon)>0$ such that among any $N_{0}$ consecutive integers there exists at least an integer $p>0$ for which

$$
\mathbf{E}\left\|X_{t+p}-X_{t}\right\|<\varepsilon, \forall t \in \mathbb{Z}_{+} .
$$

An integer $p>0$ with the above-mentioned property is called an $\varepsilon$-almost period for $X$. The collection of all $\mathbb{B}$-valued random sequences $X=\left\{X_{t}\right\}_{t \in \mathbb{Z}_{+}}$which are Bohr almost periodic in mean is then denoted by $A P\left(\mathbb{Z}_{+} ; L^{1}(\Omega ; \mathbb{B})\right)$.

The proofs of the following propositions are straightforward.

Proposition 2.2. Let $X=\left\{X_{t}, t \in \mathbb{Z}_{+}\right\}$be an $L^{1}(\Omega, \mathbb{B})$-valued almost periodic random sequence. Then, the sequence defined by $Y_{t}:=X_{t-r}$ for all $t \geq r$, for some fixed $r \in \mathbb{Z}_{+}$, is almost periodic in mean.

Definition 2.3. An $L^{1}(\Omega ; \mathbb{B})$-valued random sequence $X=\left\{X_{t}\right\}_{n \in \mathbb{Z}_{+}}$is called mean Bochner almost periodic if for every sequence $\left\{m_{k}\right\}_{k \in \mathbb{Z}_{+}} \subset \mathbb{Z}_{+}$there exists a subsequence $\left\{m_{k}^{\prime}\right\}_{k \in \mathbb{Z}_{+}}$such that $\left\{X_{t+m_{k}^{\prime}}\right\}_{k \in \mathbb{Z}_{+}}$converges (in the mean) uniformly in $t \in \mathbb{Z}_{+}$.

Following along the same arguments as in the proof of [Diagana et al. [6], Theorem 2.4, p. 241], one can show that those two notions of almost periodicity coincide.

Theorem 2.4. An $L^{1}(\Omega ; \mathbb{B})$-valued random sequence $X=\left\{X_{t}\right\}_{n \in \mathbb{Z}_{+}}$is Bochner almost periodic in mean if and only if it is Bohr almost periodic in mean.

An important and straightforward consequence of Theorem 2.4 is the next corollary, which pays a key role in the proof of our main result

Corollary 2.5. If $X_{1}=\left\{X_{t}^{1}\right\}_{t \in \mathbb{Z}_{+}}, X_{2}=\left\{X_{t}^{2}\right\}_{t \in \mathbb{Z}_{+}}, \ldots$, and $X_{N}=\left\{X_{t}^{N}\right\}_{t \in \mathbb{Z}_{+}}$are $N$ random sequences, which belong to $A P\left(\mathbb{Z}_{+} ; L^{1}(\Omega, \mathbb{B})\right)$, then for each $\varepsilon>0$ there exists $N_{0}(\varepsilon)>0$ such that among any $N_{0}(\varepsilon)$ consecutive 
integers there exists an integer $p>0$ for which

$$
\mathbf{E}\left\|X_{t+p}^{j}-X_{t}\right\|<\varepsilon
$$

for $t \in \mathbb{Z}_{+}$and for $j=1,2, \ldots, N$.

Definition 2.6. A $\mathbb{B}$-valued random sequence $X=\left\{X_{t}\right\}_{t \in \mathbb{Z}_{+}}$is said to be almost periodic in probability if for each $\varepsilon>0$, and $\eta>0$ there exists $N_{0}(\varepsilon, \eta)>0$ such that among any $N_{0}$ consecutive integers there exists at least an integer $p>0$ for which

$$
\mathbf{P}\left\{\omega \in \Omega:\left\|X_{t+p}(\omega)-X_{t}(\omega)\right\|>\varepsilon\right\}<\eta, \forall t \in \mathbb{Z}_{+} .
$$

Theorem 2.7. If $X$ is almost periodic in mean, then it is almost periodic in probability and there also exists a constant $M>0$ such that $\mathbf{E}\left\|X_{t}\right\| \leq M$ for all $t \in \mathbb{Z}_{+}$. Conversely, if $X$ is almost periodic in probability and the sequence $\left\{\left\|X_{t}\right\|, t \in \mathbb{Z}_{+}\right\}$is uniformly integrable, then $X$ is almost periodic in mean.

Let $\mathbf{k}=\{k(i)\}_{i \in \mathbb{Z}_{+}}$and denote $T_{\mathbf{k}} X_{t}(\omega):=\lim _{i \rightarrow \infty} X_{t+k(i)}(\omega)$ for each $\omega \in \Omega$ and each $t \in \mathbb{Z}_{+}$if it exists.

Definition 2.8. A $\mathbb{B}$-valued random sequence $X=\left\{X_{t}\right\}_{n \in \mathbb{Z}_{+}}$satisfies Bochner's almost sure uniform double sequence criterion if, for every pair of sequences $\left(k_{i}^{\prime}\right)$ and $\left(l_{i}^{\prime}\right)$, there exists a measurable subset $\Omega_{1} \subset \Omega$ with $\mathbf{P}\left(\Omega_{1}\right)=1$ and there exist subsequences $\mathbf{k}=\left(k_{i}\right) \subset\left(k_{i}^{\prime}\right)$ and $\mathbf{l}=\left(l_{i}\right) \subset\left(l_{i}^{\prime}\right)$ respectively, with the same indexes (independent of $\omega$ ) such that, for every $t \in \mathbb{Z}_{+}$,

$$
T_{\mathbf{k}} T_{\mathbf{l}} X_{t}(\omega)=T_{\mathbf{k}+\mathbf{1}} X_{t}(\omega), \forall \omega \in \Omega_{1} .
$$

(In this case, $\Omega_{1}$ depends on the pair of sequences $\left(k_{i}^{\prime}\right)$ and $\left(l_{i}^{\prime}\right)$.

Theorem 2.9. [1] The following properties of $X$ are equivalent:

(i) X satisfies Bochner's almost sure uniform double sequence criterion.

(ii) $X$ is almost periodic in probability.

The proof of the theorem can be seen in Bedouhene et al. [1] for instance.

Theorem 2.10. [1] If $X$ satisfies Bochner's almost sure uniform double sequence criterion and the sequence $\left\{\left\|X_{t}\right\|, t \in \mathbb{Z}_{+}\right\}$is uniformly integrable, then $X$ is almost periodic in mean.

Let $\left(\mathbb{B}_{1},\|\cdot\|_{1}\right)$ and $\left(\mathbb{B}_{2},\|\cdot\|_{2}\right)$ be Banach spaces and let $L^{1}\left(\Omega ; \mathbb{B}_{1}\right)$ and $L^{1}\left(\Omega ; \mathbb{B}_{2}\right)$ be their corresponding $L^{1}$. spaces, respectively.

Definition 2.11. A function $F: \mathbb{Z}_{+} \times L^{1}\left(\Omega ; \mathbb{B}_{1}\right) \mapsto L^{1}\left(\Omega ; \mathbb{B}_{2}\right),(t, U) \mapsto F(t, U)$ is said to be almost periodic in mean in $n \in \mathbb{Z}_{+}$uniformly in $U \in \mathbb{K}$ where $\mathbb{K} \subset L^{1}\left(\Omega ; \mathbb{B}_{1}\right)$ is a compact if for any $\varepsilon>0$, there exists a positive integer $l(\varepsilon, \mathbb{K})$ such that among any $l$ consecutive integers there exists at least a integer $p$ with the following property

$$
\mathbf{E}\|F(t+p, U)-F(t, U)\|<\varepsilon
$$

for each random variable $U \in L^{1}\left(\Omega ; \mathbb{B}_{1}\right)$ and $t \in \mathbb{Z}_{+}$.

Here again, the number $p$ will be called an $\varepsilon$-translation of $F$ and the set of all $\varepsilon$-translations of $F$ is denoted by $\varepsilon(\varepsilon, F, \mathbb{K})$.

Let $U B\left(\mathbb{Z}_{+} ; L^{1}(\Omega ; \mathbb{B})\right)$ denote the collection of all uniformly bounded $L^{1}(\Omega ; \mathbb{B})$-valued random sequences $X=$ $\left\{X_{t}\right\}_{t \in \mathbb{Z}_{+}}$. It is then easy to check that the space $U B\left(\mathbb{Z}_{+} ; L^{1}(\Omega ; \mathbb{B})\right)$ is a Banach space when it is equipped with the norm:

$$
\|X\|_{\infty}=\sup _{t \in \mathbb{Z}_{+}} \mathbf{E}\left\|X_{t}\right\| .
$$

Lemma 2.12. $A P\left(\mathbb{Z}_{+} ; L^{1}(\Omega ; \mathbb{B})\right) \subset U B\left(\mathbb{Z}_{+} ; L^{1}(\Omega ; \mathbb{B})\right)$ is a closed space. 
In view of the above, the space $A P\left(\mathbb{Z}_{+} ; L^{1}(\Omega ; \mathbb{B})\right)$ of almost periodic random sequences equipped with the sup norm $\|\cdot\|_{\infty}$ is also a Banach space.

We now state the following composition result.

Theorem 2.13. [2] Let $F: \mathbb{Z}_{+} \times L^{1}\left(\Omega ; \mathbb{B}_{1}\right) \mapsto L^{1}\left(\Omega ; \mathbb{B}_{2}\right),(t, U) \mapsto F(t, U)$ be almost periodic in mean in $t \in \mathbb{Z}_{+}$ uniformly in $U \in L^{1}\left(\Omega ; \mathbb{B}_{1}\right)$. If in addition, $F$ is Lipschitz in $U \in \mathbb{K}$, where $\mathbb{K} \subset L^{1}\left(\Omega ; \mathbb{B}_{1}\right.$ ) is compact, (That is, there exists $L>0$ such that

$$
\left.\mathbf{E}\|F(t, U)-F(t, V)\|_{2} \leq M \mathbf{E}\|U-V\|_{1} \quad \forall U, V \in L^{1}\left(\Omega ; \mathbb{B}_{1}\right), t \in \mathbb{Z}_{+}\right)
$$

then for any almost periodic random sequence $X=\left\{X_{t}\right\}_{t \in Z+}$, then the $L^{1}\left(\Omega ; \mathbb{B}_{1}\right)$-valued random sequence $Y_{t}=$ $F\left(t, X_{t}\right)$ is almost periodic in mean.

\section{Main Result}

In the rest of the paper we let $\left(\mathbb{R}_{+},|\cdot|\right),\left(\mathbb{R}^{k},\|\cdot\|_{k}\right)$ be the field of nonnegative real numbers equipped with its absolute value, the $k$-dimensional space of real numbers equipped with the Euclidean norm, which is defined by $\left\|\left(x_{1}, \ldots, x_{k}\right)\right\|_{k}=\sqrt{x_{1}^{2}+\ldots+x_{k}^{2}}$, for all $\left(x_{1}, \ldots, x_{k}\right) \in \mathbb{R}^{k}$, respectively.

We begin this section with the following crucial lemma.

Lemma 3.1. Consider the function $f: \mathbb{R}^{\tau+1} \rightarrow \mathbb{R}_{+}$defined by

$$
f\left(t, X_{t-\tau}, X_{t-\tau+1}, \ldots, X_{t-1}, X_{t}\right)=\frac{\left(1-\gamma_{t}\right) \mu K_{t} X_{t}}{\left(1-\gamma_{t}\right) K_{t}+\left(\mu-1+\gamma_{t}\right)\left(\sum_{i=1}^{\tau+1} \beta_{i} X_{t-i+1}\right)},
$$

where both $\left\{K_{t}\right\}_{t \in \mathbb{Z}_{+}}$and $\left\{\gamma_{t}\right\}_{t \in \mathbb{Z}_{+}}$belong to $A P\left(\mathbb{Z}_{+} ; L^{1}\left(\Omega ; \mathbb{R}_{+}\right)\right)$and $\mu>1$. Then

(i) $f$ is Lipschitz in the following sense:

$$
\mathbf{E}\left|f\left(t,\left(U_{t-\tau}, U_{t-\tau+1}, \ldots, U_{t-1}, U_{t}\right)\right)-f\left(t,\left(V_{t-\tau}, V_{t-\tau+1}, \ldots, V_{t-1}, V_{t}\right)\right)\right| \leq L \mathbf{E}\|U-V\|_{\tau+1}
$$

where

$$
L=\mu \sqrt{\tau+1}\left[2+\frac{1}{\beta_{1}} \sqrt{\sum_{i=1}^{\tau+1} \beta_{i}^{2}}\right]
$$

and

$$
\|U-V\|_{\tau+1}=\left(\sum_{i=1}^{\tau+1}\left|U_{t-i+1}-V_{t-i+1}\right|^{2}\right)^{1 / 2},
$$

with $\tau$ being the time delay;

(ii) If $X$ is almost periodic in mean, then the sequence

$\left\{f\left(t,\left(X_{t-\tau}, X_{t-\tau+1}, \ldots, X_{t-1}, X_{t}\right)\right)\right\}_{t \in \mathbb{Z}_{+}}$is almost periodic in mean.

Proof. To prove (i), set $A_{t}=\left(1-\gamma_{t}\right) K_{t}, B_{t}=\mu-1+\gamma_{t}$, and $C_{t}(X)=\sum_{i=1}^{\tau+1} \beta_{i} X_{t-i+1}$. Then $f$ can be written as follows:

$$
f\left(t,\left(X_{t-\tau}, X_{t-\tau+1}, \ldots, X_{t-1}, X_{t}\right)\right)=\mu \frac{A_{t} X_{t}}{A_{t}+B_{t} C_{t}(X)}
$$

for each $t \in \mathbb{Z}_{+}$.

We then have 
$\left|f\left(t, X_{t-\tau}, X_{t-\tau+1}, \ldots, X_{t-1}, X_{t}\right)-f\left(t, Y_{t-\tau}, Y_{t-\tau+1}, \ldots, Y_{t-1}, Y_{t}\right)\right|$

$=\mu A_{t}\left|\frac{X_{t}}{A_{t}+B_{t} C_{t}(X)}-\frac{Y_{t}}{A_{t}+B_{t} C_{t}(Y)}\right|$

$=\mu A_{t}\left|\frac{A_{t}\left[X_{t}-Y_{t}\right]+B_{t}\left[X_{t} C_{t}(Y)-Y_{t} C_{t}(X)\right]}{\left[A_{t}+B_{t} C_{t}(X)\right]\left[A_{t}+B_{t} C_{t}(Y)\right]}\right|$

$\leq \mu A_{t}\left|\frac{A_{t}\left[X_{t}-Y_{t}\right]+B_{t} X_{t}\left\{C_{t}(Y)-C_{t}(X)\right\}}{\left[A_{t}+B_{t} C_{t}(X)\right]\left[A_{t}+B_{t} C_{t}(Y)\right]}\right|+\mu A_{t}\left|\frac{\left.B_{t} C_{t}(X)\left[X_{t}-Y_{t}\right]\right)}{\left[A_{t}+B_{t} C_{t}(X)\right]\left[A_{t}+B_{t} C_{t}(Y)\right]}\right|$

$\leq \mu\left\{\frac{\left(A_{t}\right)^{2}}{\left[A_{t}+B_{t} C_{t}(X)\right]\left[A_{t}+B_{t} C_{t}(Y)\right]}\left|X_{t}-Y_{t}\right|+\frac{A_{t} B_{t} X_{t}}{\left[A_{t}+B_{t} C_{t}(X)\right]\left[A_{t}+B_{t} C_{t}(Y)\right]} \times\right.$

$\left.\times\left\{\sum_{i=1}^{\tau+1} \beta_{i}\left|Y_{t-i+1}-X_{t-i+1}\right|\right\}+\frac{\left.A_{t} B_{t} C_{t}(X)\left|X_{t}-Y_{t}\right|\right)}{\left[A_{t}+B_{t} C_{t}(X)\right]\left[A_{t}+B_{t} C_{t}(Y)\right]}\right\}$

But

$$
\begin{aligned}
\frac{\left(A_{t}\right)^{2}}{\left[A_{t}+B_{t} C_{t}(X)\right]\left[A_{t}+B_{t} C_{t}(Y)\right]} & \leq 1, \\
\frac{A_{t} B_{t} X_{t}}{\left[A_{t}+B_{t} C_{t}(X)\right]\left[A_{t}+B_{t} C_{t}(Y)\right]} & \leq \frac{1}{\beta_{1}}, \\
\frac{\left.A_{t} B_{t} C_{t}(X)\left|X_{t}-Y_{t}\right|\right)}{\left[A_{t}+B_{t} C_{t}(X)\right]\left[A_{t}+B_{t} C_{t}(Y)\right]} & \leq 1 .
\end{aligned}
$$

Thus,

$$
\begin{aligned}
& \mathbf{E}\left|f\left(t, X_{t-\tau}, X_{t-\tau+1}, \ldots, X_{t-1}, X_{t}\right)-f\left(t, Y_{t-\tau}, Y_{t-\tau+1}, \ldots, Y_{t-1}, Y_{t}\right)\right| \\
& \leq \mu\left\{2\left|X_{t}-Y_{t}\right|+\frac{1}{\beta_{1}}\left\{\sum_{i=1}^{\tau+1} \beta_{i}\left|Y_{t-i+1}-X_{t-i+1}\right|\right\}\right\} .
\end{aligned}
$$

Note that

$$
|| X_{j}-Y_{j} \mid \leq \sqrt{\tau+1} \sqrt{\sum_{i=1}^{\tau+1}\left|X_{t-i+1}-Y_{t-i+1}\right|^{2}} \text { for any } 1 \leq j \leq \tau+1
$$

and

$$
\sum_{i=1}^{\tau+1} \beta_{i}\left|Y_{t-i+1}-X_{t-i+1}\right| \leq\left(\sum_{i=1}^{\tau+1} \beta_{i}^{2}\right)^{1 / 2}\left(\sum_{i=1}^{\tau+1}\left|X_{t-i+1}-Y_{t-i+1}\right|^{2}\right)^{1 / 2} .
$$

Thus,

$$
\begin{aligned}
& \mathbf{E}\left|f\left(t, X_{t-\tau}, X_{t-\tau+1}, \ldots, X_{t-1}, X_{t}\right)-f\left(t, Y_{t-\tau}, Y_{t-\tau+1}, \ldots, Y_{t-1}, Y_{t}\right)\right| \\
& \quad \leq L \mathbf{E}\left\|\left(X_{t-\tau}, X_{t-\tau+1}, \ldots, X_{t-1}, X_{t}\right)-\left(Y_{t-\tau}, Y_{t-\tau+1}, \ldots, Y_{t-1}, Y_{t}\right)\right\|_{\tau+1} .
\end{aligned}
$$

To prove (ii), we use the fact that $\left\{\gamma_{t}\right\},\left\{K_{n}\right\}$, and $\left\{X_{t}\right\}$ are almost periodic in mean and make use of Corollary 2.5. We can then choose $M>0$ such that $\mathbf{E}\left|K_{t}\right|<M$ for all $t \in \mathbb{Z}_{+}$and for each $\varepsilon>0$ there exists a positive integer $N_{0}(\varepsilon)$ consecutive integers. there exists an integer $p>0$. a common $\varepsilon$-almost periodic for $\left\{\gamma_{t}\right\},\left\{K_{t}\right\}$, and $\left\{X_{t}\right\}$ such that

$\mathbf{E}\left|\gamma_{t+p}-\gamma_{t}\right| \leq \frac{\varepsilon \beta_{1}(\mu-1)^{2}}{4 \mu^{2} M}, \mathbf{E}\left|K_{t+p}-K_{t}\right| \leq \frac{\varepsilon \beta_{1}(\mu-1)}{8 \mu^{2}}, \mathbf{E}\left|X_{t+p}-X_{t}\right| \leq \frac{\varepsilon}{4 \mu}$ for all $t \in \mathbb{Z}_{+}$, and $\mathbf{E}\left|\beta_{i} X_{t+p-i+1}-\beta_{i} X_{t-i+1}\right| \leq \frac{\beta_{1} \varepsilon}{4(\tau+1) \mu}$, for each $i=1, \ldots, \tau+1$ and for all $t \in \mathbb{Z}_{+}$. 
Using the same notations as before, we now evaluate:

$$
\begin{aligned}
\left|f\left(t+p, X_{t+p-\tau}, X_{t+p-\tau+1}, \ldots, X_{t+p-1}, X_{t+p}\right)-f\left(t, X_{t-\tau}, X_{t-\tau+1}, \ldots, X_{t-1}, X_{t}\right)\right| & \\
= & \mu\left|\frac{A_{t+p} X_{t+p}}{A_{t+p}+B_{t+p} C_{t+p}(X)}-\frac{A_{t} X_{t}}{A_{t}+B_{t} C_{t}(X)}\right| \\
\leq & \mu\left|\frac{A_{t+p} X_{t+p}}{A_{t+p}+B_{t+p} C_{t+p}(X)}-\frac{A_{t+p} X_{t}}{A_{t+p}+B_{t+p} C_{t+p}(X)}\right|+\mu\left|\frac{A_{t+p} X_{t}}{A_{t+p}+B_{t+p} C_{t+p}(X)}-\frac{A_{t+p} X_{t}}{A_{t+p}+B_{t+p} C_{t}(X)}\right| \\
& +\mu\left|\frac{A_{t+p} X_{t}}{A_{t+p}+B_{t+p} C_{t}(X)}-\frac{A_{t} X_{t}}{A_{t}+B_{t} C_{t}(X)}\right| \\
\leq & \mu\left|\frac{A_{t+p}\left[X_{t+p}-X_{t}\right]}{A_{t+p}+B_{t+p} C_{t+p}(X)}\right|+\mu A_{t+p} X_{t}\left|\frac{1}{A_{t+p}+B_{t+p} C_{t+p}(X)}-\frac{1}{A_{t+p}+B_{t+p} C_{t}(X)}\right| \\
& +\mu\left|\frac{A_{t+p} X_{t}}{A_{t+p}+B_{t+p} C_{t}(X)}-\frac{A_{t} X_{t}}{A_{t}+B_{t} C_{t}(X)}\right| \\
\leq & \mu\left|X_{t+p}-X_{t}\right|+\mu \frac{A_{t+p} B_{t+p} X_{t}\left|C_{t+p}(X)-C_{t}(X)\right|}{\left(A_{t+p}+B_{t+p} C_{t+p}\right)\left(A_{t+p}+B_{t+p} C_{t}\right)}+\mu\left|\frac{X_{t} C_{t}(X)\left[A_{t+p} B_{t}-A_{t} B_{t+p}\right]}{\left(A_{t+p}+B_{t+p} C_{t}(X)\right)\left(A_{t}+B_{t} C_{t}(X)\right)}\right| \\
\leq & \mu\left|X_{t+p}-X_{t}\right|+\mu \frac{X_{t}\left|C_{t+p}(X)-C_{t}(X)\right|}{C_{t}(X)}+\mu\left|\frac{X_{t}\left[A_{t+p} B_{t}-A_{t} B_{t+p}\right]}{\left.B_{t+p} B_{t} C_{t}(X)\right)}\right|
\end{aligned}
$$

But

$C_{t}(X) \geq \beta_{1} X_{t}$.

Thus,

$$
\begin{aligned}
& \left|f\left(t+p, X_{t+p-\tau}, X_{t+p-\tau+1}, \ldots, X_{t+p-1}, X_{t+p}\right)-f\left(t, X_{t-\tau}, X_{t-\tau+1}, \ldots, X_{t-1}, X_{t}\right)\right| \\
& \leq \mu\left\{\left|X_{t+p}-X_{t}\right|+\frac{1}{\beta_{1}}\left|C_{t+p}(X)-C_{t}(X)\right|+\frac{1}{\beta_{1}}\left|\frac{A_{t+p}}{B_{t+p}}-\frac{A_{t}}{B_{t}}\right|\right\} \\
& \leq \mu\left\{\left|X_{t+p}-X_{t}\right|+\frac{1}{\beta_{1}} \sum_{i=1}^{\tau+1} \beta_{i}\left|X_{t+p-i+1}-X_{t-i+1}\right|+\frac{1}{\beta_{1}}\left|\frac{A_{t+p}}{B_{t+p}}-\frac{A_{t}}{B_{t}}\right|\right\},
\end{aligned}
$$

which in turn implies that

$\mathbf{E}\left|f\left(t+p, X_{t+p-\tau}, X_{t+p-\tau+1}, \ldots, X_{t+p-1}, X_{t+p}\right)-f\left(t, X_{t-\tau}, X_{t-\tau+1}, \ldots, X_{t-1}, X_{t}\right)\right|$.

$\leq \mu\left\{\mathbf{E}\left|X_{t+p}-X_{t}\right|+\frac{1}{\beta_{1}} \sum_{i=1}^{\tau+1} \beta_{i} \mathbf{E}\left|X_{t+p-i+1}-X_{t-i+1}\right|+\frac{1}{\beta_{1}} \mathbf{E}\left|\frac{A_{t+p}}{B_{t+p}}-\frac{A_{t}}{B_{t}}\right|\right\}$.

We now evaluate carefully $\mathbf{E}\left|\frac{A_{t+p}}{B_{t+p}}-\frac{A_{t}}{B_{t}}\right|$ using the hypothesis of independence of random sequence $\left\{\gamma_{t}\right\}_{t \in \mathbb{Z}_{+}}$.

$$
\begin{aligned}
& \mathbf{E}\left|\frac{A_{t+p}}{B_{t+p}}-\frac{A_{t}}{B_{t}}\right|=\mathbf{E}\left|\frac{\left(1-\gamma_{t+p}\right) K_{t+p}}{\mu-1+\gamma_{t+p}}-\frac{\left(1-\gamma_{t}\right) K_{t}}{\mu-1+\gamma_{t}}\right| \\
& =\mathbf{E}\left[\frac{1}{\left(\mu-1+\gamma_{t+p}\right)\left(\mu-1+\gamma_{t}\right)}\left|(\mu-1)\left[K_{t+p}-K_{t}\right]-\gamma_{n} \gamma_{t+p}\left[K_{t+p}-K_{t}\right]-(\mu-1)\left[\gamma_{t+p} K_{t+p}-\gamma_{t} K_{t}\right]+\left[\gamma_{t} K_{t+p}-\gamma_{t+p} K_{t}\right]\right|\right] \\
& =\mathbf{E}\left[\frac{1}{\left(\mu-1+\gamma_{t+p}\right)\left(\mu-1+\gamma_{n}\right)} \mid(\mu-1)\left[K_{t+p}-K_{t}\right]-\gamma_{t} \gamma_{t+p}\left[K_{t+p}-K_{n}\right]\right.
\end{aligned}
$$




$$
\begin{aligned}
& \left.-(\mu-1) K_{t+p}\left[\gamma_{t+p}-\gamma_{t}\right]+\gamma_{t}\left[K_{t+p}-K_{t}\right]+\gamma_{t}\left[K_{t+p}-K_{t}\right]-\left[\gamma_{t+p}-\gamma_{t}\right] \mid\right] \\
= & \mathbf{E} \mid \frac{\mu-1}{\left(\mu-1+\gamma_{t+p}\right)\left(\mu-1+\gamma_{t}\right)}\left[K_{t+p}-K_{t}\right]-\frac{\gamma_{t} \gamma_{t+p}}{\left(\mu-1+\gamma_{t+p}\right)\left(\mu-1+\gamma_{t}\right)}\left[K_{t+p}-K_{t}\right] \\
& -\frac{\mu-1}{\left(\mu-1+\gamma_{t+p}\right)\left(\mu-1+\gamma_{t}\right)} K_{t+p}\left[\gamma_{t+p}-\gamma_{t}\right]+\frac{(\mu-1) \gamma_{t}}{\left(\mu-1+\gamma_{t+p}\right)\left(\mu-1+\gamma_{t}\right)}\left[K_{t+p}-K_{t}\right] \\
& -\frac{1}{\left(\mu-1+\gamma_{t+p}\right)\left(\mu-1+\gamma_{t}\right)}\left[K_{t+p}-K_{t}\right]-\frac{1}{\left(\mu-1+\gamma_{t+p}\right)\left(\mu-1+\gamma_{t}\right)} K_{t}\left[\gamma_{t+p}-\gamma_{t}\right] \mid \\
\leq & \frac{1}{\mu-1} \mathbf{E}\left|K_{t+p}-K_{t}\right|+\mathbf{E}\left|K_{t+p}-K_{t}\right|+\frac{1}{\mu-1} \mathbf{E}\left|K_{t+p}\right| \mathbf{E}\left|\gamma_{t+p}-\gamma_{t}\right| \\
& +\mathbf{E}\left|K_{t+p}-K_{t}\right|+\frac{1}{\mu-1} \mathbf{E}\left|K_{t+p}-K_{t}\right|+\frac{1}{(\mu-1)^{2}} \mathbf{E}\left|K_{t}\right| \mathbf{E}\left|\gamma_{t+p}-\gamma_{t}\right| \\
\leq & \left.\frac{2 \mu}{\mu-1} \mathbf{E}\left|K_{t+p}-K_{t}\right|\right]+\frac{\mu}{(\mu-1)^{2}} M \cdot \mathbf{E}\left|\gamma_{t+p}-\gamma_{t}\right| \cdot
\end{aligned}
$$

By combining, we obtain

$$
\begin{aligned}
& \mathbf{E}\left|f\left(t+p, X_{t+p-\tau}, X_{t+p-\tau+1}, \ldots, X_{t+p-1}, X_{t+p}\right)-f\left(t, X_{t-\tau}, X_{t-\tau+1}, \ldots, X_{t-1}, X_{t}\right)\right| . \\
& \leq \mu\left\{\mathbf{E}\left|X_{t+p}-X_{t}\right|+\frac{1}{\beta_{1}} \sum_{i=1}^{\tau+1} \beta_{i} \mathbf{E}\left|X_{t+p-i+1}-X_{t-i+1}\right|+\frac{1}{\beta_{1}}\left\{\frac{2 \mu}{\mu-1} \mathbf{E}\left|K_{t+p}-K_{t}\right|+\frac{\mu}{(\mu-1)^{2}} M \cdot \mathbf{E}\left|\gamma_{t+p}-\gamma_{t}\right|\right\}\right\} \\
& \quad \leq \frac{\varepsilon}{4}+\frac{\varepsilon}{4}+\frac{\varepsilon}{4}+\frac{\varepsilon}{4}=\varepsilon .
\end{aligned}
$$

We now state our main result.

Theorem 3.2. Suppose that both random sequences $\left\{K_{t}\right\}_{t \in \mathbb{Z}_{+}}$and $\left\{\gamma_{t}\right\}_{t \in \mathbb{Z}_{+}}$belong to $A P\left(\mathbb{Z}_{+} ; L^{1}\left(\Omega ; \mathbb{R}_{+}\right)\right)$. Then, Eq.(1.1) has a unique almost periodic solution whenever

$$
\sup _{t \in \mathbb{Z}_{+}} \mathbf{E}\left|\gamma_{t}\right|<\frac{1}{L+1}
$$

where $L$ satisfies (3.1).

We now prove Theorem 3.2.

Proof. By Lemma 3.1(ii), if $\varphi \in A P\left(\mathbb{Z}_{+}, L^{1}\left(\Omega ; \mathbb{R}_{+}\right)\right.$, then $t \mapsto f(t, \varphi(t-\tau), \varphi(t-\tau+1), \ldots, \varphi(t-1), \varphi(t))$ belongs to $A P\left(\mathbb{Z}_{+}, L^{1}\left(\Omega ; \mathbb{R}_{+}\right)\right)$.

Define the nonlinear operator $\Gamma$ by setting

$$
\Gamma: A P\left(\mathbb{Z}_{+}, L^{1}\left(\Omega ; \mathbb{R}_{+}\right)\right) \rightarrow A P\left(\mathbb{Z}_{+}, L^{1}\left(\Omega ; \mathbb{R}_{+}\right)\right),
$$

where

$$
(\Gamma \varphi)(t):=\sum_{r=0}^{t-1}\left(\prod_{s=r}^{t-1} \gamma_{s}\right) f(s, \varphi(s-\tau), \varphi(s-\tau+1), \cdots, \varphi(s-1), \varphi(s)) .
$$

It is clear that $\Gamma$ is well defined. Now, let $\varphi, \psi \in A P\left(\mathbb{Z}_{+}, L^{1}\left(\Omega ; \mathbb{R}_{+}\right)\right)$having the same property as $X$ defined in Eq.(1.1). Since $\gamma_{t}, t \in \mathbb{Z}_{+}$are independent and independent of $\varphi$ and $\psi$, one can easily see that

$$
\begin{aligned}
\mathbf{E}|\Gamma \varphi(t)-\Gamma \psi(t)| & \leq \sum_{r=0}^{t-1}\left\{\left(\prod_{s=r}^{t-1} \mathbf{E}\left|\gamma_{s}\right|\right) \times\right. \\
& \times \mathbf{E} \mid f\left(r, \varphi_{r-\tau}, \varphi_{r-\tau+1}, \cdots, \varphi_{r-1}, \varphi_{r}\right) \\
& -f\left(r,\left(\psi_{r-\tau}, \psi_{r-\tau+1}, \cdots, \psi_{r-1}, \psi_{r}\right) \mid\right\},
\end{aligned}
$$


and hence letting $\beta=\sup _{n \in \mathbb{Z}_{+}} \mathbf{E}\left[\gamma_{n}\right]$ and using Lemma 3.1(i) we obtain

$$
\begin{aligned}
\sup _{t \in \mathbb{Z}_{+}} \mathbf{E}|\Gamma \varphi(t)-\Gamma \psi(t)| & \leq\left(\frac{L \beta}{1-\beta}\right) \times \\
& \times \sup _{t \in \mathbb{Z}_{+}} \mathbf{E} \|\left(\varphi_{t-\tau}, \varphi_{t-\tau+1}, \ldots, \varphi_{t-1}, \varphi_{t}\right) \\
& -\left(\psi_{t-\tau}, \psi_{t-\tau+1}, \ldots, \psi_{t-1}, \psi_{t}\right) \|_{\tau+1} .
\end{aligned}
$$

Hence,

$$
\|\Gamma \varphi-\Gamma \psi\|_{\infty} \leq \frac{L \beta}{1-\beta}\|\varphi-\psi\|_{\infty} .
$$

Obviously, $\Gamma$ is a contraction whenever $\frac{L \beta}{1-\beta}<1$. In that event, using the Banach fixed point theorem it easily follows that $\Gamma$ has a unique fixed point, $\bar{X}$, which obviously is the unique mean almost periodic solution of Eq.(1.1).

\section{References}

[1] Bedouhene, F., Mellah, O., and Raynaud de Fitte, P., Bochner- almost periodicity for stochastic processes, Stochastic Anal. Appl., 30(2) (2012):322-342.

[2] Bezandry, P. and Diagana, T., 2011. Almost periodic stochastic processes, Springer, New York.

[3] Bezandry, P., Diagana, T., and Elaydi, S., On the stochastic Beverton-Holt equation with survival rates, J. Difference Eq. Appl. 14 (2008), no. 2, pp. 175-190.

[4] Corduneanu, C., 1989. Almost periodic functions, 2nd Edition, Chelsea-New York.

[5] Diagana, T., Almost automorphic solutions to a Beverton-Holt dynamic equation with survival rate, Applied Mathematics Letters, 36 (2014), 19-24

[6] Diagana, T., Elaydi, S., and Yakubu, A-A, Population models in almost periodic environments, J. Difference Equations and Appl., 13 (2007), no. 4, 239-260.

[7] Franke, J. E. and Yakubu,A-A, Population models with periodic recruitment functions and survival rates, J. Diff. Equations Appl., 11 (2005), No. 14, 1169-1184.

[8] Han, J. and Hong, C., Almost periodic random sequences in probability, J. Math. Anal. Appl., 336 (2007), 962-974.

[9] Hong, J. and Nunez, C., The almost periodic type difference equations, Mathl. Comput. Modeling, Vol. 28 (1998), No. 12, pp. 21-31. 